\title{
A GENERALIZED METHOD FOR THE OPTIMAL DESIGN OF BEAMS UNDER FLEXURAL VIBRATION
}

\author{
Y. S. LIAO \\ Department of Mechanical Engineering, National Taiwan University, Taipei, Taiwan, \\ Republic of China
}

(Received 3 January 1992)

\begin{abstract}
A generalized method is developed for the design of a cantilever of circular cross-section in flexural vibration. The dynamic rigidity of the overhang bar can be increased if the root portion of the bar is made of high elastic modulus material while the free end portion is composed of low density material. Hence, the method developed is for a beam composed of two materials at different ends, but the method applies to a beam of a single material as well. The variational statement is written, and a set of non-linear boundary value equations are obtained. By solving these equations, the shape of the bar and the material length ratio (length of the material at root portion over total overhang length) which would result in a highest first mode natural frequency is determined. The optimal shape, material length ratio and flexural rigidity of the overhang bar composed of two materials under various constraints are discussed.
\end{abstract}

\section{INTRODUCTION}

Cantilever tools are widely used in practical manufacturing engineering. Boring bars and internal grinding mandrels are two typical examples. However, the low stiffness and low structural damping of the cantilever structures always result in the occurrence of chatter (self-excited vibration) during cutting with the use of cantilever tools.

To increase the dynamic rigidity of cantilever tools such as those used in boring operations, the so-called combination boring bar with the root and tool head portions made of different materials has been studied by Rivin [1], Rivin and Kang [2], New and $\mathrm{Au}$ [3] and Takeyama et al. [4]. On the other hand, a beam of tapered shape or of different cross-sections at the fixed and free ends is used in certain applications. Regarding the optimum tapering of cantilever, Karihaloo and Niordson used the variational approach to solve the problem [5]. Cantilever beams of rectangular cross-section having maximum fundamental bending frequency under various constraints were studied extensively by Elwany and Barr [6], and the computed results and profiles in each case were given. A generalized method for the optimum design of a cantilever beam composed of two materials is developed in this paper. From the beam equation, the variational functional is formulated for a bar composed of two materials with end mass and rotatory inertia under various constraints such as the bounds of cross-section and constant volume. The variation of the functional for the deflection and shape function of the bar gives a set of non-linear boundary value equations. By solving the set of simultaneous equations with boundary conditions by a numerical method, the bar having the specific shape and length ratio which results in the maximum fundamental natural frequency can be obtained. 


\section{ANALYSIS}

\subsection{DIFFERENTIAL EQUATIONS AND BOUNDARY CONDITIONS}

Let the vibration-resistant bar be composed of two materials as shown in Figure 1. The root portion and free end portion of the bar are made of materials 1 and 2 respectively. These two materials meet at a distance away from the clamped position. If the bar is regarded as a cantilever beam, then the classical Bernoulli-Euler beam theory is applicable. The effect of rotatory inertia of the end mass is included but that of transverse shear deformation is neglected in this analysis. The deflections, slopes, moments and shear forces should be equal in the two sides of the bar at the location where they meet. These assumptions are reasonably valid if the beam under consideration is vibrating in its lower modes, and the interface of the two materials is rigid.

Let $E_{1}$ and $\rho_{1}$ be the Young's modulus and density of material 1, while $E_{2}$ and $\rho_{2}$ are the similar quantities for material 2, and let $I(x)$ and $A(x)$ be the inertia moment and cross-section area of the bar at a location a distance $x$ from the clamped position, respectively. If the deflection at time $t$ is denoted as $U(x, t)$, then the equations of motion of the bar in flexural motion can be written as (a list of nomenclature is given in the Appendix)

$$
\begin{array}{ll}
\frac{\partial^{2}}{\partial x^{2}}\left[E_{1} I(x) \frac{\partial^{2} U(x, t)}{\partial x^{2}}\right]+\rho_{1} A(x) \frac{\partial^{2} U(x, t)}{\partial t^{2}}=0, & 0<x<L_{1}, \\
\frac{\partial^{2}}{\partial x^{2}}\left[E_{2} I(x) \frac{\partial^{2} U(x, t)}{\partial x^{2}}\right]+\rho_{2} A(x) \frac{\partial^{2} U(x, t)}{\partial t^{2}}=0, & L_{1}<x<L .
\end{array}
$$

For free vibration, in the normal mode, $U(x, t)$ is a harmonic motion of time. Let $U(x, t)=u(x) \mathrm{e}^{\mathrm{i} \omega t}$, where $\omega$ is the vibration frequency; then substituting $U(x, t)$ into equations (la) and (b) gives

$$
\begin{array}{ll}
\frac{\mathrm{d}^{2}}{\mathrm{~d} x^{2}}\left[E_{1} I(x) \frac{\mathrm{d}^{2} u(x)}{\mathrm{d} x^{2}}\right]-\rho_{1} A(x) \omega^{2} u(x)=0, & 0<x<L_{1}, \\
\frac{\mathrm{d}^{2}}{\mathrm{~d} x^{2}}\left[E_{2} I(x) \frac{\mathrm{d}^{2} u(x)}{\mathrm{d} x^{2}}\right]-\rho_{2} A(x) \omega^{2} u(x)=0, & L_{1}<x<L .
\end{array}
$$

The boundary conditions required in solving the differential equations (2a) and (2b) are (1) at the fixed end of the bar (i.e., $x=0$ ),

$$
u(0)=\mathrm{d} u(0) / \mathrm{d} x=0,
$$

and (2) at the free end of the bar (i.e., $x=L$ )

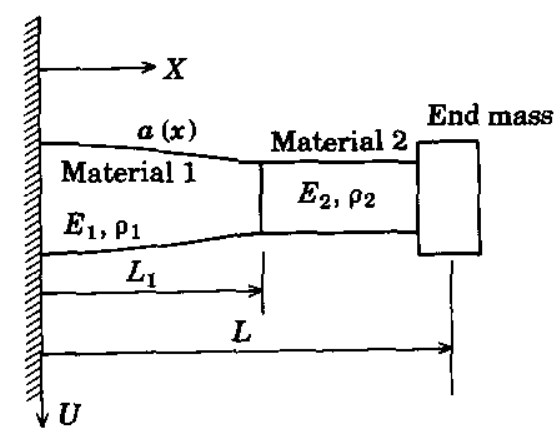

Figure 1. Model of the bar in the study. 


$$
E_{2} I(L) \frac{\mathrm{d}^{2} u(L)}{\mathrm{d} x^{2}}=J \omega^{2} \frac{\mathrm{d} u(L)}{\mathrm{d} x}, \quad \frac{\mathrm{d}}{\mathrm{d} x}\left[E_{2} I(L) \frac{\mathrm{d}^{2} u(L)}{\mathrm{d} x^{2}}\right]=-M \omega^{2} u(L) .
$$

In these equations, $J$ is the rotary inertia, and $M$ is the end mass. At the interface of the two materials (i.e., $x=L_{1}$ )

$$
\begin{gathered}
u\left(L_{1}^{-}\right)=u\left(L_{1}^{+}\right), \quad \mathrm{d} u\left(L_{1}^{-}\right) / \mathrm{d} x=\mathrm{d} u\left(L_{1}^{+}\right) / \mathrm{d} x, \\
E_{1} I\left(L_{1}^{-}\right) \frac{\mathrm{d}^{2} u\left(L_{1}^{-}\right)}{\mathrm{d} x^{2}}= \\
=E_{2} I\left(L_{1}^{+}\right) \frac{\mathrm{d}^{2} u\left(L_{1}^{+}\right)}{\mathrm{d} x^{2}}, \quad \frac{\mathrm{d}}{\mathrm{d} x}\left[E_{1} I\left(L_{1}^{-}\right) \frac{\mathrm{d}^{2} u\left(L_{1}^{-}\right)}{\mathrm{d} x^{2}}\right] \\
=\frac{\mathrm{d}}{\mathrm{d} x}\left[E_{2} I\left(L_{1}^{+}\right) \frac{\mathrm{d}^{2} u\left(L_{1}^{+}\right)}{\mathrm{d} x^{2}}\right] .
\end{gathered}
$$

For a circular bar, if the radius at a distance $x$ away from the clamped position is denoted by $r(x)$, then $A(x)=\pi r^{2}(x)$ and $I(x)=\pi r^{4}(x) / 4$. By introducing these two quantities into the equations of motion and the boundary conditions, and with the non-dimensional quantities $\mu=E_{2} / E_{1}, \quad v=\rho_{1} / \rho_{2}, \quad l=L_{1} / L, S=\mu \nu, \quad X=x / L, \quad \vec{r}^{2}=$ $\int_{0}^{L} r^{2}(x) \mathrm{d} x / L, \alpha(X)=\left[r(x) / \bar{r}^{2}\right]$ and $Y(X)=u(x) / u\left(x_{0}\right)$, where $x_{0}$ is any convenient point in the interval $0<x_{0}<L$, equations $(2 \mathrm{a})$ and $(2 \mathrm{~b})$ become

$$
\begin{gathered}
{\left[\alpha^{2}(X) Y^{\prime \prime}(X)\right]^{\prime \prime}-S B^{2} \alpha(X) Y(X)=0, \quad 0<X<l,} \\
{\left[\alpha^{2}(X) Y^{\prime \prime}(X)\right]^{\prime \prime}-B^{2} \alpha(X) Y(X)=0, \quad l<X<1 .}
\end{gathered}
$$

In these equations the prime indicates differentiation with respect to the dimensionless co-ordinate $X$. The frequency parameter $B$ is defined as $B^{2}=\left(4 \rho_{2} L^{4} \omega^{2}\right) /\left(E_{2} r^{2}\right)$.

The boundary conditions specified by equations ( $3 \mathrm{a})-(3 \mathrm{~d})$ become

$$
Y(0)=Y^{\prime}(0)=0, \quad \alpha^{2}(1) Y^{\prime \prime}(1)=B^{2} \bar{J} Y^{\prime}(1), \quad\left[\alpha^{2}(1) Y^{\prime \prime}(1)\right]^{\prime}=-B^{2} \bar{M} Y(1), \quad(6 \mathrm{a}-\mathrm{d})
$$

where $\left.\bar{J}=J / \pi \bar{r}^{2} \rho_{2} L^{3}\right)$ and $\bar{M}=M /\left(\pi \bar{r}^{2} \rho_{2} L\right)$.

The conditions imposed at the interface of the two materials become

$$
\begin{gathered}
Y\left(l^{-}\right)=Y\left(l^{+}\right), \quad Y^{\prime}\left(l^{-}\right)=Y^{\prime}\left(l^{+}\right), \\
\alpha^{2}\left(l^{-}\right) Y^{\prime \prime}\left(l^{-}\right)=\mu \alpha^{2}\left(l^{+}\right) Y^{\prime \prime}\left(l^{+}\right), \quad\left[\alpha^{2}\left(l^{-}\right) Y^{\prime \prime}\left(l^{-}\right)\right]^{\prime}=\mu\left[\alpha^{2}\left(l^{+}\right) Y^{\prime \prime}\left(l^{+}\right)\right]^{\prime} .
\end{gathered}
$$

\subsection{VARIATIONAL STATEMENT}

Multiplying equation (5a) by $Y(X)$, and equation (5b) by $\mu Y(X)$, and then integrating the resultant equations with respect to $X$ between their upper and lower bounds separately, yields

$$
\int_{0}^{l}\left(\alpha^{2} Y^{\prime \prime}\right)^{\prime \prime} Y \mathrm{~d} X-S B^{2} \int_{0}^{l} \alpha Y^{2} \mathrm{~d} X=0, \quad \mu \int_{1}^{1}\left(\alpha^{2} Y^{\prime \prime}\right)^{\prime \prime} Y \mathrm{~d} X-\mu B^{2} \int_{i}^{1} \alpha Y^{2} \mathrm{~d} X=0 .
$$

Integration by parts can be applied to the first terms of these two equations. Then, substituting the conditions specified by equations (6a)-(7d) into the sum of the two resultant equations, and a little manipulation, yields

$$
B^{2}=\frac{\int_{0}^{l} \alpha^{2}\left(Y^{\prime \prime}\right)^{2} \mathrm{~d} X+\mu \int_{l}^{1} \alpha^{2}\left(Y^{\prime \prime}\right)^{2} \mathrm{~d} X}{S \int_{0}^{1} \alpha Y^{2} \mathrm{~d} X+\mu \int_{l}^{1} \alpha Y^{2} \mathrm{~d} X+\mu \bar{M}(Y(1))^{2}+\mu J\left(Y^{\prime}(1)\right)^{2}} .
$$


The condition that the volume of the bar is kept constant at some value $Q$ in the optimum search can be expressed as

$$
Q=\int_{0}^{L} A(x) \mathrm{d} x=\int_{0}^{L} \pi r^{2}(x) \mathrm{d} x=\pi \ddot{r} L \int_{0}^{L} \frac{r^{2}(x)}{\bar{r}^{2}} \frac{\mathrm{d} x}{L}=\pi \vec{r}^{2} L \int_{0}^{1} \alpha(X) \mathrm{d} X .
$$

Since $Q$ equals the volume of the uniform bar of radius $\bar{r}$, the constraint condition becomes

$$
\int_{0}^{1} \alpha(X) \mathrm{d} X=1
$$

Let $\bar{\alpha}$ and $\alpha^{*}$ be the lower and upper bounds of the cross-section; then

$$
\alpha(X)-\bar{\alpha}=a^{2}(X), \quad \alpha^{*}-\alpha(X)=b^{2}(X),
$$

where the functions $a(X)$ and $b(X)$ are arbitrary in the non-constant radius region of the bar.

A variational technique can be used for the purpose of finding the function $\alpha(X)$ which maximizes the frequency parameter $B$ while satisfying equation (11). Let a functional be

$$
\begin{aligned}
f[Y, \alpha]= & \frac{\int_{0}^{l} \alpha^{2}\left(Y^{\prime \prime}\right)^{2} \mathrm{~d} X+\mu \int_{l}^{1} \alpha^{2}\left(Y^{\prime \prime}\right)^{2} \mathrm{~d} X}{S \int_{0}^{1} \alpha Y^{2} \mathrm{~d} X+\mu \int_{1}^{1} \alpha Y^{2} \mathrm{~d} X+\mu \bar{M}(Y(1))^{2}+\mu \bar{J}\left(Y^{\prime}(1)\right)^{2}} \\
& +\bar{\lambda}_{1}\left[\int_{0}^{1} \alpha(X) \mathrm{d} X-1\right]+\int_{0}^{1} \lambda_{2}(X)\left[\alpha(X)-\bar{\alpha}-a^{2}(X)\right] \mathrm{d} X \\
& +\int_{0}^{1} \lambda_{3}(X)\left[\alpha^{*}-\alpha(X)-b^{2}(X)\right] \mathrm{d} X,
\end{aligned}
$$

where $\bar{\lambda}_{1}, \lambda_{2}(X)$ and $\lambda_{3}(X)$ are Lagrange multipliers. $\bar{\lambda}_{1}$ is an unknown constant, but $\lambda_{2}(X)$ and $\lambda_{3}(X)$ are known functions. The functional $f$ is stationary with respect to small variations of the variables $Y, \alpha, a(X)$ and $b(X)$. Let $\delta$ [] be the derivative of a quantity with respect to a particular variable. Then, with a little manipulation, and with use of equations (6a)-(7d), the first of the Euler stationary conditions, which is $\delta f[Y, \alpha]$ with respect to $Y$, can be reduced to

$$
\int_{0}^{l}\left[\left(\alpha^{2} Y^{\prime \prime}\right)^{\prime \prime}-S B^{2} \alpha Y\right] \delta Y \mathrm{~d} X+\int_{l}^{1} \mu\left[\left(\alpha^{2} Y^{\prime \prime}\right)^{\prime \prime}-B^{2} \alpha Y\right] \delta Y \mathrm{~d} X=0 .
$$

This can be expressed as

$$
\left(\alpha^{2} Y^{\prime \prime}\right)^{\prime \prime}-S B^{2} \alpha Y=0, \quad 0<X<l, \quad\left(\alpha^{2} Y^{\prime \prime}\right)^{\prime \prime}-B^{2} \alpha Y=0, \quad l<X<1 .
$$

It should be noted that equations (15a) and (15b) are the same as equations (5a) and (5b).

Similarly, the stationary conditions of the Euler equation with respect to $\alpha$ can be reduced to

$$
\begin{gathered}
2 \alpha\left(Y^{\prime \prime}\right)^{2}-S B^{2} Y^{2}-\lambda_{1}^{*}+\lambda_{2}^{*}(X)-\lambda_{3}^{*}(X)=0, \\
2 \mu \alpha\left(Y^{\prime \prime}\right)^{2}-\mu B^{2} Y^{2}-\lambda_{1}^{*}+\lambda_{2}^{*}(X)-\lambda_{3}^{*}(X)=0,
\end{gathered}
$$

where $\lambda_{1}^{*}=-\bar{\lambda}_{1} \beta, \lambda_{2}^{*}(X)=\lambda_{2}(X) \beta, \lambda_{3}^{*}(X)=\lambda_{3}(X) \beta$ and

$$
\beta=S \int_{0}^{l} \alpha Y^{2} \mathrm{~d} X+\mu \int_{l}^{1} \alpha Y^{2} \mathrm{~d} X+\mu \bar{M}(Y(1))^{2}+\mu \bar{J}\left(Y^{\prime}(1)\right)^{2} .
$$


The stationary conditions of the Euler equation with respect to $a(X)$ and $b(X)$ separately are

$$
-2 \lambda_{2}(X) a(X)=0, \quad-2 \lambda_{3}(X) b(X)=0 .
$$

From equation (17a) either $\lambda_{2}(X)=0$ or $a(X)=0$, and from equation (17b) either $\lambda_{3}(X)=0$ or $b(X)=0$. If $a(X)=0$ then $\alpha=\bar{\alpha}$, while if $b(X)=0$ then $\alpha=\alpha^{*}$. Hence these conditions cannot hold simultaneously. In the non-constant radius region $\lambda_{2}(X)=$ $\lambda_{3}(X)=0$, and equations (16a) and (16b) are reduced to

$$
2 \alpha\left(Y^{\prime \prime}\right)^{2}-S B^{2} Y^{2}-\lambda_{1}^{*}=0, \quad 0<X<l, \quad 2 \mu \alpha\left(Y^{\prime \prime}\right)^{2}-\mu B^{2} Y^{2}-\lambda_{1}^{*}=0, \quad l<X<1 .
$$

If $Y$ and $Y^{\prime \prime}$ are normalized by $\sqrt{\left|\lambda_{1}^{*}\right|}$, then in the non-uniform cross-section region the governing equations (equations (15a), (15b), (18a) and (18b)) for $0<X<l$ become

$$
\left(\alpha^{2} Y^{\prime \prime}\right)^{\prime \prime}-S B^{2} \alpha Y=0, \quad 2 \alpha\left(Y^{\prime \prime}\right)^{2}-S B^{2} Y^{2}-\lambda=0,
$$

and for $l<X<1$ they become

$$
\left(\alpha^{2} Y^{\prime \prime}\right)^{\prime \prime}=B^{2} \alpha Y=0, \quad 2 \mu \alpha\left(Y^{\prime \prime}\right)^{2}-\mu B^{2} Y^{2}-\lambda=0,
$$

where, in equations $(19 a)-(20 b), \lambda$ takes on the values \pm 1 only.

\section{NUMERICAL INTEGRATION SOLUTION}

The optimal shape and length ratio of a two-material combination boring bar can be obtained by solving equations (11) and (19a)-(20b). If a function $D(X)$ is defined as

$$
D(X)=\int_{0}^{X} \alpha\left(X^{*}\right) \mathrm{d} X^{*},
$$

then equation (11) is equivalent to $D(0)=0$, and $D(1)=1$. Moreover, equations (19a) and (20a) can be written as

$$
\begin{aligned}
& Y^{\prime}=F, \quad F^{\prime}=\mu G / \alpha^{2}, \quad G^{\prime}=H, \quad H^{\prime}=v B^{2} \alpha Y, \quad 0<X<l, \\
& Y^{\prime}=F, \quad F^{\prime}=G / \alpha^{2}, \quad G^{\prime}=H, \quad H^{\prime}=B^{2} \alpha Y, \quad l<X<1 .
\end{aligned}
$$

The problem of finding the radius distribution and the optimal length ratio for a maximum frequency bar becomes that of solving the set of the above two first order equations, together with the calculation of equations (19b) and (20b).

Specifically, the bar is divided into $N$ (integer) equal spans along its length. Suppose that the intersection is at a certain point. For this intersection point, the unknown parameters $B, Y^{\prime \prime}(0)$ and $\left[\alpha^{2} Y^{\prime \prime}\right]_{X=0}^{\prime}$ are guessed first, and equations (22a) and (22b) are integrated with conditions given by equations $(6 a),(6 b)$ and $(7 a)-(7 d)$. The profile of the bar is computed by using equations $(19 \mathrm{~b})$ and $(20 \mathrm{~b})$. The computed end conditions are checked with the boundary conditions specified by equations $(6 \mathrm{c})$ and $(6 \mathrm{~d})$. If they do not match to within a certain small allowance, the initial guess $B$ is kept unchanged while the initial guesses $Y^{\prime \prime}(0)$ and $\left[\alpha^{2} Y^{\prime \prime}\right]_{X=0}^{\prime}$ are modified by the adjoint method [7], and the procedures are repeated. Once the boundary conditions are satisfied, equation (21) is evaluated. For an arbitrary guess of $B$, the boundary condition $D(0)=0$ and $D(1)=1$ cannot be satisfied simultaneously. The frequency parameter $B$ is then adjusted and all the procedures are repeated. The foregoing procedures are applied to different intersection points, and the length ratio and the profile associated with the largest value of $B$ are the final optimal solutions. 
TABLE 1

Optimal $B$ and $X_{c}$ for a cantilever beam having rectangular and circular cross-sections for various $\bar{\alpha}$

\begin{tabular}{cccccc}
\hline & \multicolumn{2}{c}{ Elwany and Barr [6] } & \multicolumn{2}{c}{ Present } \\
\cline { 2 - 3 } $\bar{\alpha}$ & $B$ & $X_{c}$ & & $X_{c}$ \\
\hline 1.00 & 3.51608 & 0 & 3.51632 & 0 \\
0.90 & 4.21175 & 0.36739 & 4.21125 & 0.36875 \\
0.80 & 4.77914 & 0.47831 & 4.77900 & 0.48000 \\
0.70 & 5.34360 & 0.55295 & 5.34299 & 0.55500 \\
0.60 & 5.94304 & 0.61090 & 5.94249 & 0.61250 \\
0.50 & 6.61051 & 0.65974 & 6.60750 & 0.66125 \\
0.40 & 7.39892 & 0.70336 & 7.38125 & 0.70500 \\
0.30 & 8.41342 & 0.74400 & 8.33000 & 0.74750 \\
\hline
\end{tabular}

\section{VERIFICATION}

A computer program based on the previous described procedures was written, and two cases were studied to ascertain if the proposed method and the computer program are correct.

\subsection{CANTILEVER MADE OF SINGLE MATERIAL}

In the first case a uniform cross-section cantilever which is made up of a single material but is without an end mass is studied. It is desired to find the shape of the bar that will result in a maximum fundamental natural frequency. To apply the method stated in the paper, the same coefficients of the materials are input to the program for the two portions of the compound bar. The optimal shapes of the bar under various cross-section constraints are determined. The computed profiles are shown in Figure 2. The beam has a tapered shape in the fixed end region, while the cross-section in the free end region remains unchanged. The corresponding beam with a rectangular cross-section of constant side ratio has been studied by Elwany and Barr [6]. For comparison purposes, the corresponding frequency parameters and the length ratio of the corner (i.e., the position at which the cross-section starts to change from uniform to tapered shape) of both beams, denoted by $X_{c}$, under various cross-section constraints are given in Table 1 . It can be seen from Table 1 that the two approaches yield almost the same results. It is also noted that for $\bar{\alpha}=0.8$ the frequency parameter can be increased by a factor of $1.36(4.78 / 3.52)$ as compared with that of a uniform cross-section bar $(\bar{\alpha}=1$ in the table). The corner is at a length ratio of 0.48 , and the dimensionless radius is 1.24 at the clamped position.

\subsection{OVERHANG BAR COMPOSED OF TWO MATERIALS}

A compound bar with the two sections made of different materials but with unity upper and lower bounds of the cross-section (i.e., uniform cross-section), and with a mass attached at the free end, was investigated. The following five combinations of the materials were considered [1]:
(a) $E_{1}=5.5 \times 10^{5} \mathrm{MPa}, \gamma_{1}=16$;
$E_{2}=0.7 \times 10^{5} \mathrm{MPa}, \gamma_{2}=2.7 ;$
(b) $E_{1}=3.5 \times 10^{5} \mathrm{MPa}, \gamma_{1}=18$;
$E_{2}=0.7 \times 10^{5} \mathrm{MPa}, \gamma_{2}=2.7$;
(c) $E_{1}=2 \cdot 1 \times 10^{5} \mathrm{MPa}, \gamma_{1}=7 \cdot 8$;
$E_{2}=0.7 \times 10^{5} \mathrm{MPa}, \gamma_{2}=2.7$
(d) $E_{1}=5.5 \times 10^{5} \mathrm{MPa}, \gamma_{1}=16$;
$E_{2}=2.1 \times 10^{5} \mathrm{MPa}, \gamma_{2}=7 \cdot 8$
(e) $E_{1}=3.5 \times 10^{5} \mathrm{Mpa}, \gamma_{1}=18$;
$E_{1}=2.1 \times 10^{5} \mathrm{MPa}, \gamma_{2}=7.8$. 


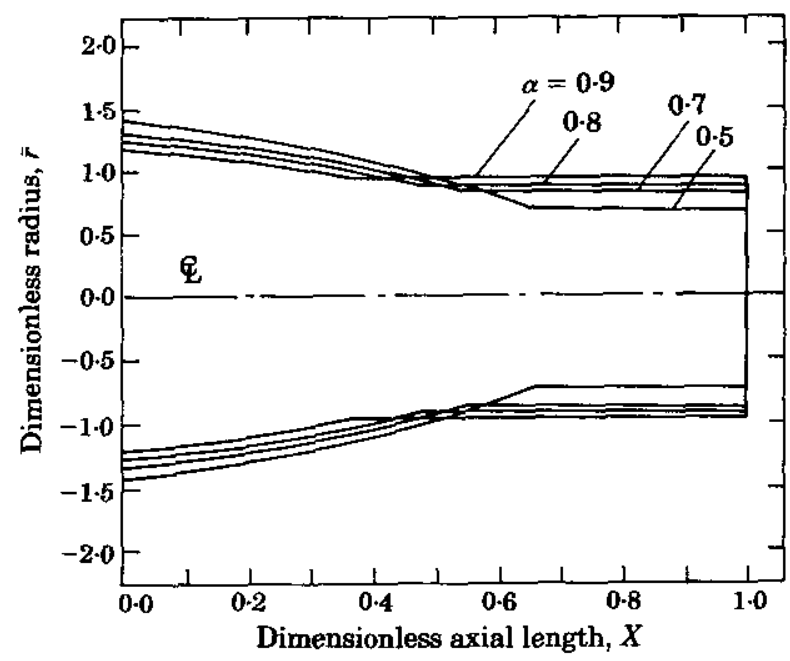

Figure 2. Computed profiles for maximum first mode bending frequency for various lower bounds $\bar{\alpha}$.

The materials corresponding to the above $E$ and $\gamma$ are as follows: aluminum $\left(E=0.7 \times 10^{5} \mathrm{MPa}, \gamma=2.7\right)$, steel $\left(E=2.1 \times 10^{5} \mathrm{MPa}, \gamma=7.8\right)$, heavy machinable tungsten alloy $\left(E=3.5 \times 10^{5} \mathrm{MPa}, \gamma=18\right)$, and sintered carbide $\left(E=5.5 \times 10^{5} \mathrm{MPa}, \gamma=16\right)$.

For bars with a length of $160 \mathrm{~mm}$, a diameter of $18.5 \mathrm{~mm}$ and an end mass of $0.0215 \mathrm{Kg}$ the results are given in Figure 3 . The same combination bars but with each bar having a chamber at the free end for the implementation of a damper have been studied by Rivin [1]. Due to the difference in the structure of the bar, these two results show small discrepancies. However, the tendency and order of the natural frequencies of bars $a, b$ and $c$, and $d$ and $e$ are the same. This comparison, together with the one given in the previous section, may also indicate that the proposed method is valid, and that the computer program is correct.

From Figure 3 it is obvious that the combination bars under study have a greater dynamic rigidity than that of the single material bar. Furthermore, the higher the elastic

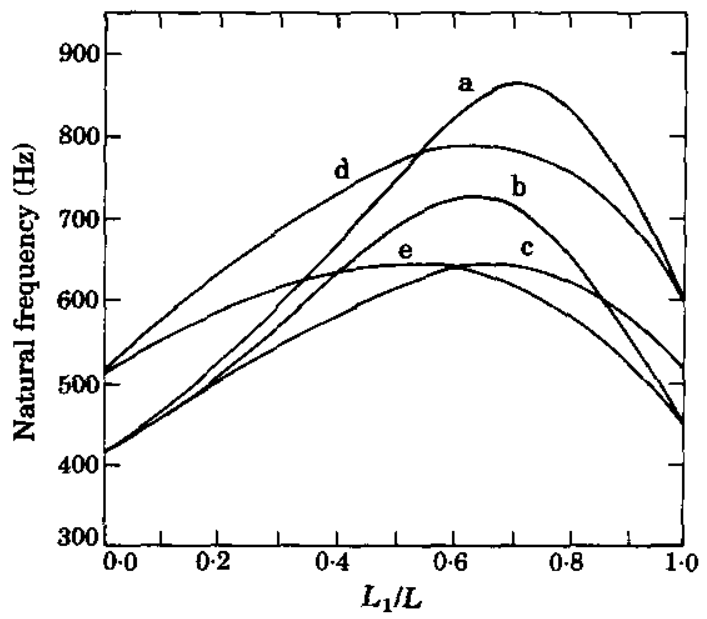

Figure 3. Natural frequency vs. length ratio for various combination boring bars having uniform cross-section. 


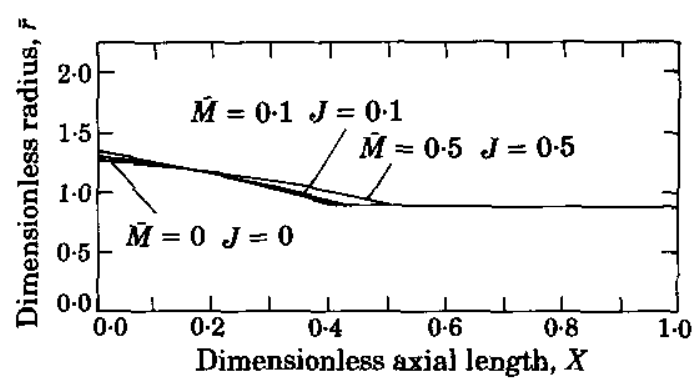

Figure 4. The optimal profile (only half the profile is shown) of the sintered carbide-aluminum combination boring bar under $\bar{\alpha}=0.8$ and various end mass constraints.

modulus of the material in the fixed end portion, and the lower the density of the material in the free end portion, the greater the fundamental natural frequency of the bar. For a bar composed of sintered carbide and aluminum, the optimal material length ratio (denoted as $X_{m}$ in Table 2 , to follow) is about 0.72 , and its fundamental natural frequency is $864 \mathrm{~Hz}$, which is 1.33 times that of the bar made of sintered carbide, and 2.07 times that of the bar made of aluminum.

\section{THE COMBINATION BAR WITH OPTIMAL SHAPE}

For illustration purposes only a bar composed of sintered carbide and aluminum is used to show the variation of the dynamic rigidity due to the change of the cross-section of the overhang bar. The radius distribution for the lower bound of $\bar{\alpha}=0.8$ under various end mass constraints is shown in Figure 4. The numerical results for the frequency parameter (B), material length ratio $\left(X_{m}\right)$, dimensionless radius at the fixed and free end portions (denoted by $\bar{r}_{1}, \bar{r}_{2}$, respectively), and upper and lower bounds corner length ratios $\left(\left(X_{c}\right)_{1}\right.$ and $\left.\left(X_{c}\right)_{2}\right)$ are summarized in Table 2 . The shape is similar to that obtained in section 4.1. However, the corner with no end mass is at a length ratio (denoted by $\left(X_{c}\right)_{2}$ in the table) of 0.41 , and it is different from that obtained in section 4.1. The length ratio for the specified two combination materials is 0.62 . The frequency parameter is found to be 10.45 . From this figure and table it can be readily seen that as the end mass is increased the corner shifts toward the free end, and the portion of high modulus material is increased together with a reduction of frequency parameter. For comparison purposes, the frequency parameter for an over-

TABLE 2

Optimal $B$ and associated design parameters of the sintered carbide-aluminum combination boring bar under $\bar{\alpha}$ and various constraints

\begin{tabular}{ccccccc}
$\bar{M}, J$ & $B$ & $\bar{r}_{1}\left(\alpha^{*}\right)$ & $\bar{r}_{2}(\bar{\alpha})$ & $X_{m}$ & $\left(X_{c}\right)_{1}$ & $\left(X_{c}\right)_{2}$ \\
\hline 0,0 & 10.45 & $1.33(--)$ & $0.90(0.8)$ & 0.62 & - & 0.41 \\
& 9.56 & $1.14(1.3)$ & $0.90(0.8)$ & 0.63 & 0.34 & 0.46 \\
$0.1,0.1$ & 8.86 & $1.30(-)$ & $0.90(0.8)$ & 0.65 & - & 0.44 \\
& 8.37 & $1.14(1.3)$ & $0.90(0.8)$ & 0.66 & 0.32 & 0.49 \\
$0.5,0.5$ & 6.01 & $1.25(--)$ & $0.90(0.8)$ & 0.83 & - & 0.51 \\
& 5.93 & $1.14(1.3)$ & $0.90(0.8)$ & 0.84 & 0.28 & 0.53 \\
\hline
\end{tabular}




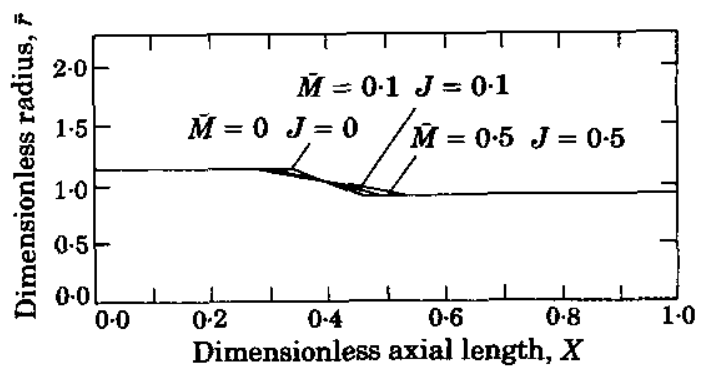

Figure 5. The optimal profile (only half the profile is shown) of the sintered carbide - aluminum combination boring bar under $\hat{\alpha}=0.8$ and $\alpha^{*}=1.3$ and various end mass constraints.

hang bar composed of the same materials with uniform cross-section but without end mass was computed, and is 6.65. This value is 1.57 times less than that of the bar in which the cross-section is allowed to be varied.

In addition to the lower bound of $\bar{\alpha}=0.8$, if the bar also has an upper bound, for example with $\alpha^{*}=1 \cdot 3$, the results for the same end mass constraints as discussed previously are shown in Figure 5 and Table 2. From Table 2 it is observed that the material length ratio is further increased while the frequency parameter would be reduced further. It can also be seen from Figure 5 that between $\left(X_{c}\right)_{1}$ and $\left(X_{c}\right)_{2}$ the bar possesses a tapered shape. When the end mass is increased, the radius distribution varies in such a manner that $\left(X_{c}\right)_{1}$ is decreased and $\left(X_{c}\right)_{2}$ is increased, the radius distribution varies in such a manner that $\left(X_{c}\right)_{1}$ is decreased and $\left(X_{c}\right)_{2}$ is increased.

\section{CONCLUSIONS}

A generalized method to solve a set of non-linear boundary value equations for the problem stated in the paper has been established. By using this method the optimal shapes and length ratios of an overhang bar composed of two materials under various constraints can be determined, and their performance can be evaluated. When there are upper, or lower, or both bounds constraints, only part of the length, instead of the whole length of the bar, has a tapered shape.

\section{REFERENCES}

1. E. I. RIvIN 1983 Proceedings of NAMRC-XI, 403-407. A chatter-resistant cantilever boring bar.

2. E. I. RIVIN and H. L. KANG 1989 Annals CIRP 38/1, 377-380. Improving dynamic performance of cantilever boring bars.

3. R. W. NEW and Y. H. J. Au 1980 Transactions of the American Society of Mechanical Engineers, Journal of Mechanical Design 102, 611-618. "Chatter-proof" overhang boring bar-stability criterion and design procedures for a new type of damped boring bar.

4. H. Takeyama, N. Lijima, N. Nishiwaki and K. Komoto 1984 annals CIRP 33/1, 249-252. Improvement of dynamic rigidity of tool holder by applying high-damping material.

5. B. L. KaRIHALOO and F. I. NIORDSON 1973 Journal of Optimization Theory and Application 11, 638-685. Optimum design of vibrating cantilevers.

6. M. H. S. ElWANY and A. D. S. BARR 1983 Journal of Sound and Vibration 88, 175-195. Optimal design of beams under flexural vibration.

7. S. M. ROBeR TS and J. S. SHIPMAN 1972 Two-Point Boundary Value Problems: Shooting Methods. New York: American Elsevier. 


\section{APPENDIX: NOMENCLATURE}

$A(x)$ $a(X), b(X)$ $B$

$D(X)$

$E_{1}, E_{2}$

$f[Y, \alpha]$

$I(x)$

$J$

$\bar{J}$

$l$

$L$

$L_{1}$

M

$\bar{M}$

$Q$

$r(x)$

$\bar{r}$

$S$

$u(x), U(x, t)$

$x$

$X$

$Y(X)$

$\bar{\alpha}, \alpha^{*}$

$\alpha(X)$

$\delta[]$

$\lambda_{1}$

$\lambda_{2}(X), \lambda_{3}(X)$

$\mu$

$v$

$\omega$

$\rho_{1}, \rho_{2}$ cross-sectional area of the bar at location $x$

arbitrary function

frequency parameter

volume function of the bar

elastic modulus of the fixed, and free, portions

functional

inertial moment of cross-section

rotatory inertia

dimensionless rotatory inertia

$L_{1} / L$ dimensionless intersection of two materials

total length of the bar

length of the fixed portion

end mass

dimensionless end mass

volume of the bar

radius of the cross-section at location $x$

mean cross-section radius

$\mu v$

deflection of the bar

axial co-ordinate

dimensionless axial co-ordinate

dimensionless deflection

lower, upper bound of cross-section

dimensionless area of cross-section (shape function) derivative of a quantity

Lagrange multiplier (constant)

Lagrange multipliers (function)

$E_{2} / E_{1}$

$\rho_{1} / \rho_{2}$

natural frequency

density of the fixed, and free, portions 